\title{
Incidence and risk factors of delirium in post-anaesthesia care unit
}

Yuhe Ke ${ }^{1}{ }_{\text {MBBS }}$, Sophia $\underline{\text { Chew }}^{1}{ }_{\text {FANZCA }}$, Edwin Seet ${ }^{2}$ MMed (Anaes), Wan Yi Wong ${ }^{3}{ }_{\text {MMed (Anaes) }}$, Vera Lim ${ }^{3}{ }_{\text {MMed (Anaes) }}$, Nelson Chua ${ }^{3}$ MMed (Anaes), Jinbin Zhang ${ }^{3}$ MMed (Anaes), Beatrice Lim ${ }^{3}{ }_{\text {MMed (Anaes), Vanessa Chua }}{ }^{4}$ Bsc (Hon), Ne-Hooi Will $\underline{\text { Loh }}{ }^{4}$ FFICM, Lian Kah $\underline{\text { Ti }}{ }^{4}$ MMed (Anaes)

\begin{abstract}
Introduction: Post-anaesthesia care unit (PACU) delirium is a potentially preventable condition that results in a significant long-term effect. In a multicentre prospective cohort study, we investigate the incidence and risk factors of postoperative delirium in elderly patients undergoing major non-cardiac surgery.

Methods: Patients were consented and recruited from 4 major hospitals in Singapore. Research ethics approval was obtained. Patients older than 65 years undergoing non-cardiac surgery $>2$ hours were recruited. Baseline perioperative data were collected. Preoperative baseline cognition was obtained. Patients were assessed in the post-anaesthesia care unit for delirium 30-60 minutes after arrival using the Nursing Delirium Screening Scale (Nu-DESC).

Results: Ninety-eight patients completed the study. Eleven patients $(11.2 \%)$ had postoperative delirium. Patients who had PACU delirium were older ( $74.6 \pm 3.2$ versus $70.6 \pm 4.4$ years, $P=0.005$ ). Univariate analysis showed those who had PACU delirium are more likely to be ASA 3 (63.6\% vs $31.0 \%, P=0.019)$, had estimated glomerular filtration rate (eGFR) of $<60 \mathrm{~mL} / \mathrm{min} / 1.73 \mathrm{~m}^{2}(36.4 \%$ vs $10.6 \%, P=0.013)$, higher $\mathrm{HbA1C}$ value $(7.8 \pm 1.2$ vs $6.6 \pm 0.9, P=0.011)$, raised random blood glucose $(10.0 \pm 5.0 \mathrm{mmol} / \mathrm{L}$ vs $6.5 \pm 2.4 \mathrm{mmol} / \mathrm{L}, P=0.0066)$, and moderate-severe depression $(18.2 \%$ vs $1.1 \%$, $P=0.033$ ). They are more likely to stay longer in hospital (median 8 days [range 4-18] vs 4 days [range 2-8], $P=0.049$ ). Raised random blood glucose is independently associated with increased PACU delirium on multivariate analysis.

Conclusion: PACU delirium is common in elderly patients with risks factors presenting for major surgery.
\end{abstract}

Ann Acad Med Singap 2022;51:87-95

Keywords: Geriatrics, major non-cardiac surgery, postoperative delirium

\section{INTRODUCTION}

Post-anaesthesia care unit (PACU) delirium is defined as a disorder in thought processes that affect cognition in terms of memory, comprehension and attention. ${ }^{1}$ It has a strong association with postoperative delirium, which is present in up to $45 \%$ of patients after surgery. ${ }^{2-5}$ PACU is a wide-reaching problem, and especially prevalent in elderly patients. Postoperative delirium directly affects clinical outcomes such as hospital length of stay and increased 30-day mortality. ${ }^{6}$ Up to $10 \%$ of these patients may develop long-term neurocognitive deficits with diminished quality of life, and pose a tremendous socioeconomic burden on family and caregivers. ${ }^{7,8}$

Despite its importance, PACU delirium remains under-diagnosed in the perioperative setting. The lack of awareness and established biomarkers could potentially contribute to increased morbidity and mortality in these vulnerable patients. Despite many screening and assessment tools ${ }^{3}$ being readily available, few hospitals have institutionalised monitoring protocols. One such scoring tool is the Nursing Delirium Screening Scale

\footnotetext{
${ }^{1}$ Division of Anaesthesiology and Perioperative Medicine, Singapore General Hospital, Singapore

${ }^{2}$ Department of Anaesthesia, Khoo Teck Puat Hospital, Singapore

${ }^{3}$ Department of Anaesthesia, Tan Tock Seng Hospital, Singapore

${ }^{4}$ Department of Anaesthesia, National University Health System, Singapore

Correspondence: A/Prof Lian Kah Ti, Department of Anaesthesia, National University Health System, 5 Lower Kent Ridge Road, Singapore 119074.

Email: lian_kah_ti@nuhs.edu.sg
} 


\section{CLINICAL IMPACT}

\section{What is New}

- This study is among the first to examine the incidence of delirium in the post-anaesthesia care unit (PACU) in Singapore.

- Among elderly patients who underwent a major non-cardiac surgery, the incidence of delirium in the PACU was $11.4 \%$.

\section{Clinical Implications}

- PACU delirium doubled the length of hospital stay and trended towards a $55 \%$ increase in hospitalisation cost.

- Future studies should investigate measures to reduce the incidence of delirium in these patients.

(Nu-DESC). It is a nurse-led, validated, quick and easy screening tool that can be performed within 2 minutes. It has a sensitivity and specificity of $98 \%$ and $92 \%$, respectively, in postoperative patients with a score of $\geq 2$ being indicative of delirium. ${ }^{3}$

With an increasingly ageing population requiring surgery, the problem of PACU delirium is set to increase. It is crucial to understand the risk factors and contributors to PACU delirium so that strategies to reduce it can be adopted. In line with this new focus, several guidelines have been promulgated to address this issue, including the Brain Health Initiative ${ }^{9}$ and the Safe Brain Initiative. ${ }^{10}$ Previous pilot study in our local population has found that the incidence of PACU delirium is up to $6.2 \%$ in the elderly population, ${ }^{11}$ while other studies found up to $25 \%$ prevalence of PACU delirium. ${ }^{12}$ Despite the high prevalence rate, our lack of understanding of the risk factors that predispose patients to PACU delirium makes it difficult for the formulation of an evidence-based guideline for prevention of delirium. The recent American Society for Enhanced Recovery and Perioperative Quality Initiative Joint Consensus Statement on Postoperative Delirium Prevention ${ }^{13}$ has recommendations that are mainly based on study in Caucasian population, and PACU delirium in Asian population has not been well studied.

Thus, we aimed to do a pragmatic observational study using $\mathrm{Nu}$-DESC to understand the incidence of PACU delirium in patients aged 65 years and older after major non-cardiac surgery in Singapore and determine its associated risk factors.

\section{METHODS}

The pragmatic observational study was conducted in 4 major hospitals in Singapore (National University Hospital, Khoo Teck Puat Hospital, Singapore General Hospital and Tan Tock Seng Hospital). Research ethics approval was obtained from the Domain Specific Review Board (DSRB Reference number 2019/00703). Written consent was obtained prior to enrollment into the study.

Patients aged 65 years and above presenting for noncardiac surgery that was expected to last longer than 2 hours were recruited in the anaesthetic preoperative consultation clinic. Exclusions included patients undergoing neurosurgical procedures and those undergoing surgery performed under local anaesthesia. The patients who fulfilled these criteria were invited to participate in a series of questionnaires involving screening for cognitive impairment with Montreal Cognitive Assessment (MoCA) score, ${ }^{14}$ depression with Patient Health Questionnaire-9 (PHQ-9) score, ${ }^{15}$ frailty with Frailty Index for Elders (FIFE) ${ }^{16}$ and nutritional screening. ${ }^{17}$ Other demographic data and perioperative parameters were collected. The research assistants administering the questionnaires were trained and certified for MoCA. All blood test results collected were based on the preoperative requirements for each patient based on individual surgical and anaesthesiologist's decisions. Each hospital was targeted to recruit 30 patients and the target for the study was to recruit 120 patients. No additional blood tests were taken for the purpose of the study.

Intraoperative details such as surgical discipline, type of anaesthesia and duration of surgery were collected. The type of surgery was classified as low, intermediate, or high risk based on the European Society of Cardiology and European Society of Anaesthesiology non-cardiac surgery risk score. ${ }^{18}$ Postoperative hypotension was defined as having a systolic blood pressure of below $90 \mathrm{mmHg}$ for more than 5 minutes.

Postoperatively, patients were assessed for PACU delirium at 30-60 minutes upon arrival in PACU using $\mathrm{Nu}$-DESC. Nu-DESC evaluates delirium based on observation of the following 5 features: (1) disorientation, (2) inappropriate behaviour, (3) inappropriate communication, (4) illusions/hallucinations and (5) psychomotor retardation. Each item was scored based on its severity from 0 to 2 . A score of $\geq 2$ was classified as a positive score for PACU delirium. ${ }^{3}$ Other postoperative variables such as postoperative hypotension (systolic blood pressure of below $90 \mathrm{mmHg}$ for more than 5 minutes.), hypothermia (core temperature $<35$ degree Celsius), desaturations ( $<92 \%$ requiring supplemental oxygen), 
postoperative nausea and vomiting, highest pain score and aggressive behaviours were collected during the PACU stay.

A follow-up phone call was conducted on postoperative day 30 to assess outcomes of the patient, including falls at home and unplanned hospital readmission. Questionnaire on 10 signs of delirium was conducted in the same setting over the phone. Data on hospital length of stay and cost of hospitalisation were also obtained for analysis.

Sample size calculation was not done for this study. All data were entered into a statistical software program, with analysis, statistical computing and visualisations carried out with SPSS Statistics software for Windows, Version 25.0 (IBM Corp, Armonk, US) and R environment version 1.2.1335 (The R Foundation for Statistical Computing, Vienna, Austria). For continuous variables, mean and standard deviation (SD) were presented, and the Student's t-test was used to test the mean differences between the groups. For categorical variables, the chi-square test was used to compare the proportions between the groups. Risk factors with a $P$ value of $<0.10$ were fitted into a multivariate logistic regression model to determine the independent predictors for PACU delirium. The effect size was reported as an odds ratio (OR) and its 95\% confidence interval (CI). To prevent multicollinearity, all factors included in the multivariable analysis were run through variance inflation factors (VIF) and were only included if VIF was $<5$.

In an exploratory analysis attempt to find the optimum preoperative capillary blood glucose cutoffs, Youden's index was identified (highest value of specificity + sensitivity -1$)$. The detailed analysis can be found in the Supplementary Materials (Appendix in the online version of this article).

\section{RESULTS}

A total of 122 patients were recruited. Twenty-four were excluded from the study due to voluntary withdrawal $(n=3)$, cancelled operations $(n=6)$, the operation being less than 2 hours $(\mathrm{n}=8)$, patients going to intensive care unit postoperatively $(n=2)$ and patients who could not be followed up due to COVID-19 restrictions at the time of data collection $(n=5)$. Fig. 1 shows the Consolidated Standards of Reporting Trials (CONSORT) diagram.

Of the 98 patients who were included in the final analysis, 11 had a Nu-DESC score of $\geq 2$. The rate of PACU delirium was $11.2 \%$. The mean age of patients who had PACU delirium was higher than those without (74.6 \pm 3.2 years versus $70.6 \pm 4.4$ years, $P=0.005$ ) (Table 1). The baseline demographics between the 2

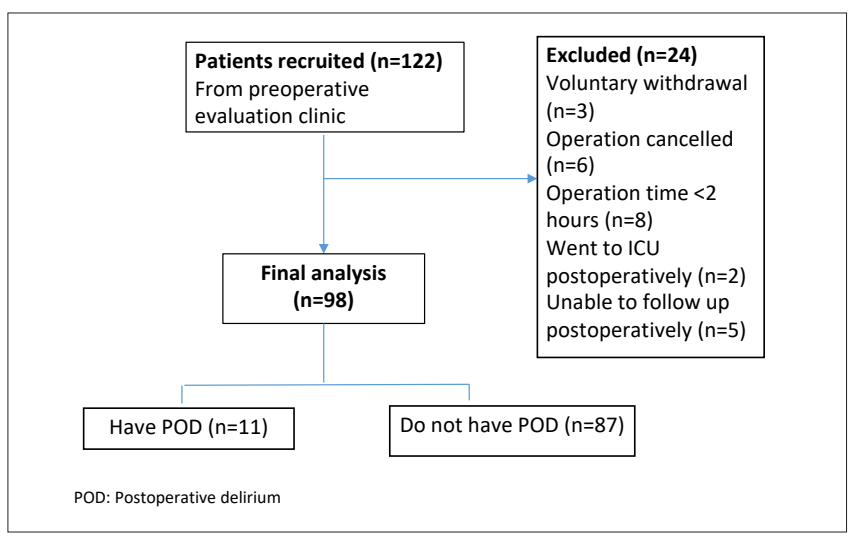

Fig. 1. Consolidated Standards of Reporting Trials (CONSORT) diagram.

groups were otherwise similar in sex, body mass index, ethnicity, and education levels. Those who had PACU delirium are more likely to be American Society of Anesthesiologists (ASA) 3 (63.6\% vs $31.0 \%, P=0.019)$, have impaired estimated glomerular filtration rate (eGFR) of $<60 \mathrm{~mL} / \mathrm{min} / 1.73 \mathrm{~m}^{2}(36.4 \%$ vs $10.6 \%$, $P=0.013)$, have higher $\mathrm{HbA1C}$ value $(7.8 \pm 1.2 \mathrm{mmol} / \mathrm{L}$ vs $6.6 \pm 0.9 \mathrm{mmol} / \mathrm{L}, P=0.011$ ), raised random blood glucose $(10.0 \pm 5.0 \mathrm{mmol} / \mathrm{L}$ vs $6.5 \pm 2.4 \mathrm{mmol} / \mathrm{L}, P=0.0066)$, and moderate to severe depression $(18.2 \%$ vs $1.1 \%, P$ $=0.033)$ (Tables 2-4).

$\mathrm{HbA} 1 \mathrm{C}$ and random blood glucose were measured based on institutional protocol. Two of the institutions routinely do random blood glucose as part of the renal panel screen. The final number included in the HbA1C analysis was 31 while 58 was included in the random blood glucose analysis.

Multivariable regression analysis shows that preoperative random blood glucose was a significant risk factor for PACU delirium (OR 1.34 95\% Cl 1.03-1.73, $P=0.027$ ) (Table 5). Fig. 2 presents the violin plot showing the distribution of preoperative random blood glucose. Eight patients had elevated random blood glucose of $>11.0 \mathrm{mmol} / \mathrm{L}$, and all of them were diabetic. In an exploratory analysis, it was found that a preoperative random blood glucose cut-off of $9.6 \mathrm{mmol} / \mathrm{L}$ is the most accurate in the association with PACU delirium (area under curve $=0.710$, specificity 0.9 , sensitivity 0.4 ) (Appendix in online Supplementary Materials).

The method of anaesthesia did not make a difference to rates of PACU delirium $(P=0.516)$, but surgeries that are deemed to be high risks contributed to PACU delirium $(36.4 \%$ vs $3.4 \%)$. The use of different anaesthetic medications such as opioids, midazolam, neuromuscular blockers, ketamine, dexmedetomidine and propofol did not contribute to a difference in PACU delirium risk. The duration of time where bispectral index (BIS) was $<30$ was not a risk factor for PACU delirium. 
Table 1. Univariate analysis of preoperative risk factors associated with post-anaesthesia care unit delirium

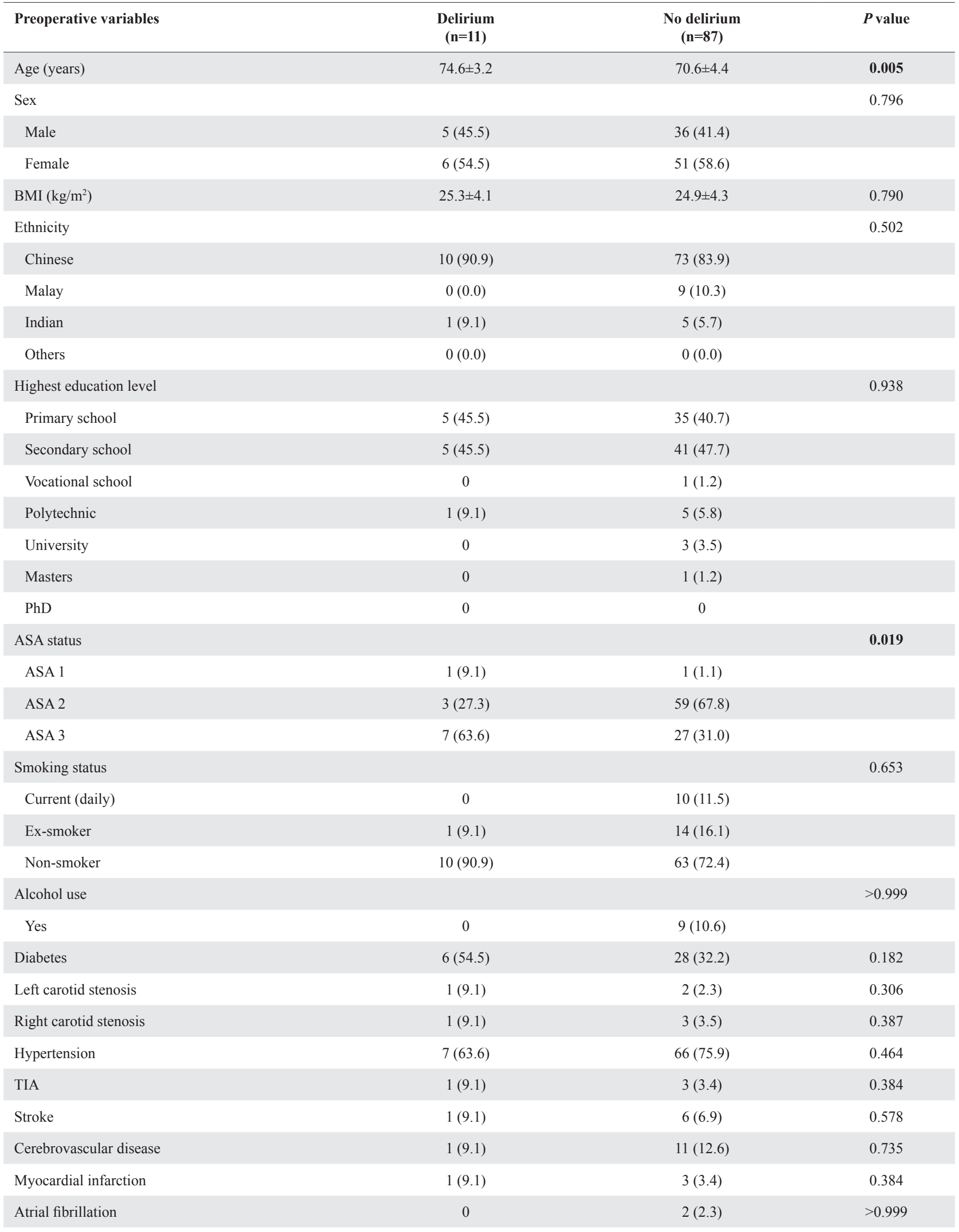


Table 1. Univariate analysis of preoperative risk factors associated with post-anaesthesia care unit delirium (Cont'd)

\begin{tabular}{|c|c|c|c|}
\hline Preoperative variables & $\begin{array}{c}\text { Delirium } \\
(\mathrm{n}=11)\end{array}$ & $\begin{array}{c}\text { No delirium } \\
\quad(n=87)\end{array}$ & $P$ value \\
\hline Asthma / COPD & 0 & $5(5.7)$ & $>0.999$ \\
\hline OSA & $2(18.2)$ & $2(2.3)$ & 0.062 \\
\hline Malignancy & $6(54.5)$ & $33(38.4)$ & 0.341 \\
\hline Opioids & $2(18.2)$ & $5(5.7)$ & 0.131 \\
\hline Antiarrhythmics & $4(36.4)$ & $13(14.9)$ & 0.077 \\
\hline Anticoagulants & $1(9.1)$ & $8(9.2)$ & 0.991 \\
\hline Statins & $6(54.5)$ & $46(52.9)$ & 0.917 \\
\hline Steroids & 0 & $2(2.3)$ & $>0.999$ \\
\hline Falls in last 3 months & 0 & $6(6.9)$ & 0.369 \\
\hline Haemoglobin $(\mathrm{g} / \mathrm{dL})$ & $14.3 \pm 3.6$ & $12.6 \pm 1.6$ & 0.135 \\
\hline $\mathrm{HbA1c}(\%)(\mathrm{n}=31)$ & $7.8 \pm 1.2$ & $6.6 \pm 0.9$ & 0.011 \\
\hline Random blood glucose $(\mathrm{mmol} / \mathrm{L})(\mathrm{n}=58)$ & $10.0 \pm 5.0$ & $6.5 \pm 2.4$ & 0.0066 \\
\hline Serum albumin $(\mu \mathrm{mol} / \mathrm{dL})$ & $41.7 \pm 1.5$ & $39.9 \pm 4.7$ & 0.061 \\
\hline PHQ-9 & & & 0.033 \\
\hline None to mild depression & $9(81.8)$ & $86(98.9)$ & \\
\hline Moderate to severe depression & $2(18.2)$ & $1(1.1)$ & \\
\hline STOP-Bang & & & 0.084 \\
\hline Low risk (score of 0-2) & $8(72.7)$ & $74(85.1)$ & \\
\hline Intermediate risk (score of 3-4) & $2(18.2)$ & $13(14.9)$ & \\
\hline High risk (score of 5-8) & $1(9.1)$ & 0 & \\
\hline Nutritional screening & & & 0.158 \\
\hline No nutritional risk (score of $0-2$ ) & $8(72.7)$ & $59(83.1)$ & \\
\hline Moderate malnutrition risk (score of 3-4) & $3(27.3)$ & $6(8.5)$ & \\
\hline Severe malnutrition risk (score of 5-9) & 0 & $6(8.5)$ & \\
\hline eGFR (MDRD calculation) $(\mathrm{n}=94)$ & & & 0.013 \\
\hline Less than $30 \mathrm{~mL} / \mathrm{min} / 1.73 \mathrm{~m}^{2}$ & 0 & 0 & \\
\hline 30 to $60 \mathrm{~mL} / \mathrm{min} / 1.73 \mathrm{~m}^{2}$ & $4(36.4)$ & $8(10.6)$ & \\
\hline More than $60 \mathrm{~mL} / \mathrm{min} / 1.73 \mathrm{~m}^{2}$ & $7(63.6)$ & $75(72.4)$ & \\
\hline $\mathrm{MoCA}$ & & & 0.542 \\
\hline Score less than 26 & $6(54.5)$ & $39(44.8)$ & \\
\hline Score 26 or more & $5(45.5)$ & $48(55.2)$ & \\
\hline FIFE & & & 0.067 \\
\hline No risk & $1(9.1)$ & $32(26.8)$ & \\
\hline At risk and frailty & $10(90.9)$ & $55(63.2)$ & \\
\hline
\end{tabular}

Student's t-test used for continuous variable

Chi-square test used for discrete variable

Mean $( \pm \mathrm{SD})$ or number (proportions)

Bold $P$ values are significant

ASA: American Society of Anesthesiologists; BMI: body mass index; COPD: chronic obstructive pulmonary disease; eGFR: estimated glomerular filtration rate; FIFE: Frailty Index for Elders; HbA1c: haemoglobin A1c; MDRD: modification of diet in renal disease; MoCA: Montreal Cognitive Assessment; OSA: obstructive sleep apnea; PHQ-9: Patient Health Questionnaire-9; TIA: transient ischaemic attack; STOP-Bang: Snoring history, Tired during the day, Observed stop breathing while sleep, high blood Pressure, BMI more than $35 \mathrm{~kg} / \mathrm{m}^{2}$, Age more than 50 years, Neck circumference more than $40 \mathrm{~cm}$ and male Gender 
Postoperative hypotension was a significant association with PACU delirium (18.2\% vs $1.2 \%, P=0.033)$. Patients who had PACU delirium had a significant longer length of stay in hospital (median 8 days [range $4-18$ ] vs 4 days [range $2-8$ ], $P=0.049)$. There was a trend towards increased hospitalisation costs (SGD28,124.74 $\pm 11,633.13$ vs SGD18,251.62 $\pm 11,677.13$, $P=0.051)$.

\section{DISCUSSION}

The incidence of PACU delirium in the elderly surgical population of Singapore at $11.2 \%$ was lower compared to those found in other studies. ${ }^{12}$

A variety of factors were examined in this study, including preoperative risk screening for dementia and depression. Although it has been postulated that preoperative dementia is a risk factor for PACU delirium, the MoCA cognitive screening test ${ }^{14}$ did not generate any significant association with PACU delirium. This is congruent with findings in Austin et al., ${ }^{19}$ where preoperative cognitive impairment was not associated with postoperative delirium when delirium was measured within 24-72 hours after surgery. The hypothesis that postoperative delirium may worsen preexisting cognitive decline $^{20}$ is also not supported by data from our study.

While the MoCA score did not show any association with PACU delirium, moderate to severe depression could have a potential relationship. Studies screening for postoperative delirium geriatrics depression scale found that even different components of depression are differentially predictive of PACU delirium, for instance, behavioural inactivity. ${ }^{21}$ The identification and optimisation of elderly patients at risk of these behaviours may reduce the risk of PACU delirium.

Table 2. Univariate analysis of intraoperative risk factors associated with post-anaesthesia care unit delirium

\begin{tabular}{|c|c|c|c|}
\hline Intraoperative variables & $\begin{array}{c}\text { Delirium } \\
(n=11)\end{array}$ & $\begin{array}{l}\text { No delirium } \\
\quad(n=87)\end{array}$ & $P$ value \\
\hline Surgical risk & & & 0.003 \\
\hline Low and intermediate & $7(63.6)$ & $84(96.6)$ & \\
\hline High & $4(36.4)$ & $3(3.4)$ & \\
\hline Type of anaesthesia & & & 0.151 \\
\hline $\begin{array}{l}\text { GA } \\
\text { RA }\end{array}$ & $\begin{array}{c}11(100.0) \\
0\end{array}$ & $\begin{array}{l}73(83.9) \\
14(16.1)\end{array}$ & \\
\hline Neuromuscular blockade & & & 0.248 \\
\hline Yes (reversed/atropine) & $1(9.1)$ & $4(4.7)$ & \\
\hline Yes (reversed/glycopyrrolate) & $8(72.7)$ & $47(54.7)$ & \\
\hline No & 0 & $19(22.1)$ & \\
\hline Opioid (fentanyl) & $6(54.5)$ & $57(65.5)$ & 0.474 \\
\hline Opioid (morphine) & $8(72.7)$ & $54(62.1)$ & 0.490 \\
\hline Opioid (others) & $7(63.6)$ & $46(42.4)$ & 0.182 \\
\hline Midazolam & $1(9.1)$ & $6(6.9)$ & 0.790 \\
\hline Propofol & $11(100.0)$ & $80(92.0)$ & 0.329 \\
\hline Dexmedetomidine & 0 & $1(1.1)$ & $>0.999$ \\
\hline Ketamine & $1(9.1)$ & $5(5.8)$ & 0.671 \\
\hline Blood transfusion & $1(9.1)$ & $3(3.5)$ & 0.387 \\
\hline BIS <30 (AUC: unit min) & $46.7 \pm 88.9$ & $33.0 \pm 141.8$ & 0.779 \\
\hline
\end{tabular}

Student's t-test used for continuous variable

Chi-square test used for discrete variable

Mean $( \pm \mathrm{SD})$ or number (proportions)

Bold $P$ value is significant

AUC: area under curve; BIS: bispectral index; GA: general anaesthesia; RA: regional anaesthesia 
Table 3. Univariate analysis of postoperative risk factors associated with post-anaesthesia care unit delirium

\begin{tabular}{|c|c|c|c|}
\hline Postoperative variables & $\begin{array}{c}\text { Delirium } \\
(n=11)\end{array}$ & $\begin{array}{l}\text { No delirium } \\
\quad(n=87)\end{array}$ & $P$ value \\
\hline Highest pain score & $2.1 \pm 2.6$ & $1.9 \pm 2.2$ & 0.748 \\
\hline PONV & $1(9.1)$ & $5(5.7)$ & 0.663 \\
\hline Hypothermia & 0 & $5(5.7)$ & $>0.999$ \\
\hline Hypotension & $2(18.2)$ & $1(1.2)$ & 0.033 \\
\hline Uncooperative towards staff & 0 & 0 & - \\
\hline Refusal to take medications & 0 & 0 & - \\
\hline Aggressive/Confused behaviour & 0 & 0 & - \\
\hline Psychiatric referral & 0 & 0 & - \\
\hline Pulling out tubes & 0 & 0 & - \\
\hline Wound breakdown & $1(9.1)$ & $1(1.1)$ & 0.213 \\
\hline Surgical infection & $1(9.1)$ & $1(1.1)$ & 0.213 \\
\hline
\end{tabular}

Student's t-test used for continuous variable

Chi-square test used for discrete variable

Mean $( \pm \mathrm{SD})$ or number (proportions)

Bold $P$ value is significant

PONV: postoperative nausea and vomiting

Table 4. Univariate analysis of post-anaesthesia care unit delirium and postoperative outcomes

\begin{tabular}{|c|c|c|c|}
\hline Postoperative variables & $\begin{array}{c}\text { Delirium } \\
(n=11)\end{array}$ & $\begin{array}{l}\text { No delirium } \\
\quad(n=87)\end{array}$ & $P$ value \\
\hline Length of stay, days (median) & $8(4-18)$ & $4(2-8)$ & 0.049 \\
\hline Final discharge destination & & & 0.438 \\
\hline Home & $9(81.8)$ & $78(89.7)$ & \\
\hline Community hospital & $2(18.2)$ & $9(10.3)$ & \\
\hline Acute care hospital & 0 & 0 & \\
\hline Mortality & 0 & 0 & \\
\hline Hospitalisation cost, SGD (mean) & $28124.74 \pm 11633.13$ & $18251.62 \pm 11677.132$ & 0.051 \\
\hline Hospital readmission within POD30 & $1(10.0)$ & $5(6.0)$ & 0.621 \\
\hline Falls at home within POD30 & 0 & $3(3.7)$ & $>0.999$ \\
\hline 10 signs of dementia & -1 & -9 & 0.497 \\
\hline
\end{tabular}

Student's t-test used for continuous variable

Chi-square test used for discrete variable

Mean $( \pm$ SD) or number (proportions)

Bold $P$ value is significant

POD: Postoperative day 
Table 5. Multivariable regression analysis of perioperative risk factors associated with postoperative delirium

\begin{tabular}{lccc}
\hline Risk factors & P value & Odds ratio & 95\% confidence interval \\
\hline eGFR & & & Lower \\
\hline Random blood glucose & 0.977 & 1.337 & 1.034 \\
Postoperative hypotension & 0.027 & & 1.727 \\
\hline
\end{tabular}

eGFR: estimated glomerular filtration rate

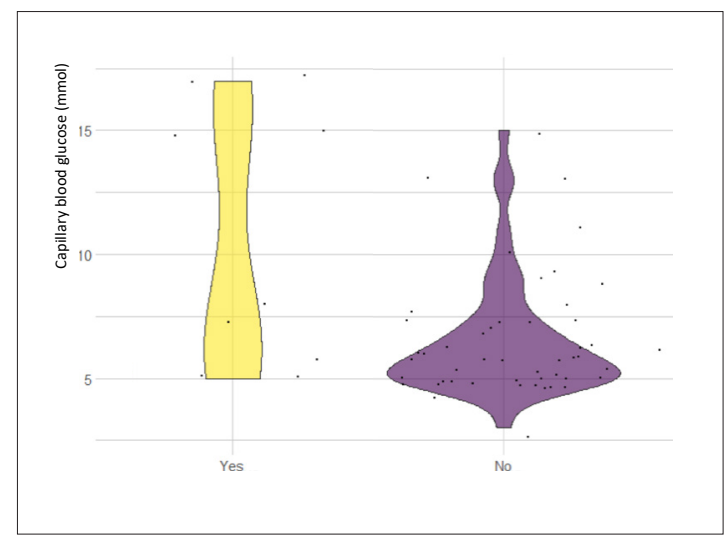

Fig. 2. Violin plot showing the distribution of preoperative random blood glucose.

The incidence of PACU delirium was not found to be different with the various anaesthesia techniques performed. Similarly, the latest American Society for Enhanced Recovery and Perioperative Quality Initiative Joint Consensus Statement on Postoperative Delirium Prevention has stated that there is no current evidence to suggest that the use of different anaesthetic agents or techniques such as central neuraxial blockade would reduce PACU delirium. ${ }^{13}$ Concurring with the findings in the Electroencephalography Guidance of Anesthesia to Alleviate Geriatric Syndromes (ENGAGES) trial, the amount of time where BIS values were between 30 and 40 intraoperatively was not associated with increased incidence of PACU delirium ${ }^{22}$ (Table 2).

While there are a few modifiable risk factors that have been associated with PACU delirium, preoperative glucose control has emerged as one potential area for optimisation. Preoperative $\mathrm{HbA} 1 \mathrm{C}$ and random blood glucose were associated with PACU delirium in our study. Lin et al. similarly found that high glycaemic variability increases the risk of PACU delirium in acute aortic dissection patients, ${ }^{23}$ while Heymann et al. also found that patients with hyperactive delirium had higher mean blood glucose than the non-hyperactive delirium patients. ${ }^{24}$
As a simple carbohydrate, the effect of glucose on the body is significant. Neurons are especially sensitive to glucose levels as they use glucose as a primary source of energy. ${ }^{25}$ Hyperglycaemia has been postulated to cause oxidative stress and neuronal damage, which may explain the association found in this study.

However, overzealous correction and tight control of perioperative blood glucose may be detrimental in vulnerable geriatric patients. Keulen et al. demonstrated that hypoglycaemia is also associated with increased delirium incidence. ${ }^{26}$

Exploratory analysis showed that preoperative random glucose of $>9.5 \mathrm{mmol} / \mathrm{L}$ may be associated with increased risk of PACU delirium, with a specificity of 0.90 and sensitivity of 0.40 . This could potentially provide a target during preoperative optimisation in elective surgery. There is hence a window of opportunity for anaesthesiologists to participate in the reduction of PACU delirium.

Patients with PACU delirium are associated with a significant increase in length of stay in hospital postoperatively in elective surgical patients $(P=0.049)$ with a median of 8 days vs 4 days. The increase in length of stay also resulted in a trend towards higher hospitalisation cost of approximately SGD10,000 $(P=0.051$, SGD28,124 $\pm 11,633$ vs SGD18,251 $\pm 11,677)$. This, however, should be interpreted in the context of patients with delirium were more likely to be elderly and of higher ASA status.

In our study population, only $31(31.6 \%)$ patients had preoperative $\mathrm{HbA} 1 \mathrm{C}$ taken, and 58 patients had random blood glucose $(59.2 \%)$. This is due to different institutional protocols across the 4 hospitals and thresholds to perform preoperative glucose control monitoring. The study is therefore limited by missing data. It highlights, however, the importance of having routine perioperative glucose monitoring for vulnerable patients. This was set up to be a pilot study and sample size calculation was not done. We cannot exclude a type 2 error for potential predictors of PACU delirium. 


\section{CONCLUSION}

PACU delirium is common (11.2\%) in elderly Asian patients $\geq 65$ years old presenting for major surgery and is associated with modifiable risk factors such as preoperative random blood glucose. Patients who had PACU delirium utilised more healthcare resources. Appropriate intervention and prevention may potentially reduce healthcare costs and improve patients' quality of life post-surgery.

\section{Disclosure}

The project is sponsored in part by Medtronic APAC under its External Research Program. Consumables such as bispectral index (BIS) monitor strips were provided for patients to facilitate the collection of intraoperative BIS data, and a total of SGD2,000 was supported for patient reimbursement.

\section{Acknowledgements}

We would like to extend our gratitude to the nurses and clinical research associates at various hospitals who had helped with data collection and acquisition.

\section{REFERENCES}

1. Bryson GL, Wyand A. Evidence-based clinical update: general anesthesia and the risk of delirium and postoperative cognitive dysfunction. Can J Anaesth 2006;53:669-77.

2. Radtke FM, Franck M, MacGuill M, et al. Duration of fluid fasting and choice of analgesic are modifiable factors for early postoperative delirium. Eur J Anaesthesiol 2010;27:411-6.

3. Radtke FM, Franck M, Schust S, et al. A comparison of three scores to screen for delirium on the surgical ward. World J Surg 2010;34:487-94

4. Winter A, Steurer MP, Dullenkopf A. Postoperative delirium assessed by post anesthesia care unit staff utilizing the Nursing Delirium Screening Scale: a prospective observational study of 1000 patients in a single Swiss institution. BMC Anesthesiol 2015;15:184.

5. Sciard D, Cattano D, Hussain M, et al. Perioperative management of proximal hip fractures in the elderly: the surgeon and the anesthesiologist. Minerva Anestesiol 2011;77:715-22.

6. Sharma PT, Sieber FE, Zakriya KJ, et al. Recovery room delirium predicts postoperative delirium after hip-fracture repair. Anesth Analg 2005;101:1215-20

7. Gaudreau J-D, Gagnon P, Harel F, et al. Fast, systematic, and continuous delirium assessment in hospitalized patients: the nursing delirium screening scale. J Pain Symptom Manage 2005;29:368-75.

8. Radtke FM, Franck M, Schneider M, et al. Comparison of three scores to screen for delirium in the recovery room. Br J Anaesth 2008;101:338-43.

9. Mahanna-Gabrielli E, Schenning KJ, Eriksson LI, et al. State of the clinical science of perioperative brain health: report from the American Society of Anesthesiologists Brain Health Initiative Summit 2018. Br J Anaesth 2019;123:464-78.
10. Aldecoa C, Bettelli G, Bilotta F, et al. European Society of Anaesthesiology evidence-based and consensus-based guideline on postoperative delirium. Eur J Anaesthesiol 2017;34:192-214.

11. Ke Y, Chew S, Seet E, et al. Risk factors of post-anaesthesia care unit delirium in patients undergoing non-cardiac surgery in Singapore. Singapore Med J 2021. doi:10.11622/smedj.2021129

12. Wu J, Gao S, Zhang S, et al. Perioperative risk factors for recovery room delirium after elective non-cardiovascular surgery under general anaesthesia. Perioper Med (Lond) 2021;10:3.

13. Hughes CG, Boncyk CS, Culley DJ, et al. American Society for Enhanced Recovery and Perioperative Quality Initiative Joint Consensus Statement on Postoperative Delirium Prevention. Anesth Analg 2020;130:1572-90.

14. Nasreddine ZS, Phillips NA, Bédirian V, et al. The Montreal Cognitive Assessment, MoCA: a brief screening tool for mild cognitive impairment. J Am Geriatr Soc 2005;53:695-9.

15. Costantini L, Pasquarella C, Odone A, et al. Screening for depression in primary care with Patient Health Questionnaire-9 (PHQ-9): A systematic review. J Affect Disord 2021;279:473-83.

16. Tocchi C, Dixon J, Naylor M, et al. Development of a frailty measure for older adults: the frailty index for elders. J Nurs Meas 2014;22:223-40.

17. Lim S-L, Tong C-Y, Ang E, et al. Development and validation of 3-Minute Nutrition Screening (3-MinNS) tool for acute hospital patients in Singapore. Asia Pac J Clin Nutr 2009;18:395-403.

18. Norderud K, Egholm G, Thim T, et al. Validation of the European Society of Cardiology and European Society of Anaesthesiology non-cardiac surgery risk score in patients treated with coronary drug-eluting stent implantation. Eur Heart J Qual Care Clin Outcomes 2019;5:22-7.

19. Austin CA, O'Gorman T, Stern E, et al. Association Between Postoperative Delirium and Long-term Cognitive Function After Major Nonemergent Surgery. JAMA Surg 2019;154:328-34.

20. Vlisides P, Avidan M. Recent Advances in Preventing and Managing Postoperative Delirium. F1000Res 2019;8:F1000 Faculty Rev-607.

21. Smith PJ, Attix DK, Weldon BC, et al. Depressive Symptoms and Risk of Postoperative Delirium. Am J Geriatr Psychiatry 2016; 24:232-8.

22. Wildes TS, Mickle AM, Ben Abdallah A, et al. Effect of Electroencephalography-Guided Anesthetic Administration on Postoperative Delirium Among Older Adults Undergoing Major Surgery: The ENGAGES Randomized Clinical Trial. JAMA 2019;321:473-83.

23. Lin Y-J, Lin L-Y, Peng Y-C, et al. Association between glucose variability and postoperative delirium in acute aortic dissection patients: an observational study. J Cardiothorac Surg 2021;16:82.

24. Heymann A, Sander M, Krahne D, et al. Hyperactive delirium and blood glucose control in critically ill patients. J Int Med Res 2007;35:666-77.

25. Scheen AJ. Central nervous system: a conductor orchestrating metabolic regulations harmed by both hyperglycaemia and hypoglycaemia. Diabetes Metab 2010;36(Suppl 3):S31-8.

26. van Keulen K, Knol W, Belitser SV, et al. Glucose variability during delirium in diabetic and non-diabetic intensive care unit patients: A prospective cohort study. PLoS One 2018;13:e0205637. 